\title{
Intramuscular hemangioma in the zygomaticus minor muscle: a case report and literature review
}

\author{
Sung Jae Wee ${ }^{1}$, \\ Myong Chul Park ${ }^{1}$, \\ Chan Min Chung', \\ Seung Wan Tak ${ }^{2}$ \\ ${ }^{1}$ Department of Plastic and \\ Reconstructive Surgery, Hallym \\ University Sacred Heart Hospital, \\ Hallym University College of Medicine, \\ Anyang; ${ }^{2}$ TN Plastic and Reconstructive \\ Surgery Clinic, Seoul, Korea
}

\begin{abstract}
Intramuscular hemangioma is a rare vascular benign proliferation that can occur within any muscle, particularly in the trunk and extremities. In the head and neck region, the masseter muscle is most commonly involved, followed by the periorbital and sternocleidomastoid muscles. Diagnosing intramuscular hemangioma is challenging because there are no characteristic symptoms; instead, magnetic resonance imaging is the best imaging modality to diagnose these lesions. Complete surgical resection is the treatment of choice, although the local recurrence rate is high. Herein, we report a rare case of intramuscular hemangioma located in the zygomaticus minor muscle, which is related to smiling and usually runs along the orbicularis oculi muscle. Distinguishing or separating these two muscles is challenging. However, based on the muscle vector of the midface and radiological findings, the two muscles were successfully separated. The zygomaticus minor was cut very slightly to approach to the lesion and the muscle fibers were split to excise it. A follow-up examination revealed no nerve damage or muscle dysfunction at 4 weeks postoperatively. This rare case may serve as a reference for managing intramuscular hemangioma in the head and neck region.
\end{abstract}

Keywords: Facial muscles / Hemangioma / Muscle fibers / Zygoma

\section{INTRODUCTION}

Intramuscular hemangioma (IMH) is a vascular benign proliferation that can occur within any muscle, particularly in the trunk and extremities [1,2]. IMH is an uncommon lesion, accounting for fewer than $1 \%$ of all hemangiomas [3]. Head and neck IMH most commonly involves the masseter muscle, followed by the periorbital and sternocleidomastoid muscles [2]. Clinically, IMH presents as a mass with slow growth, intermittent pain, and no spontaneous decrease in size. In the absence of symptomatic relief, complete surgical resection is needed, and magnetic resonance imaging (MRI) is the best imaging

\footnotetext{
Correspondence: Myong Chul Park

Department of Plastic and Reconstructive Surgery, Hallym University Sacred

Heart Hospital, Hallym University College of Medicine, 22 Gwanpyeong-ro

170beon-gil, Dongan-gu, Anyang 14068, Korea

E-mail: mpark@hallym.or.kr

Received March 15, 2021 / Revised April 13, 2021 / Accepted April 20, 2021
}

modality for evaluating IMH [3]. This paper presents a rare case of IMH located in the zygomaticus minor muscle (ZMi) that was successfully excised.

\section{CASE REPORT}

A 46-year-old woman with a fixed, non-tender, round, and protruding huge mass on her right zygoma was referred to our outpatient clinic. The diameter of the mass was approximately $2.2 \times 2.1 \mathrm{~cm}$, and she stated that it had been present for approximately 1 year, which was accompanied by a drastic change in size in the last 4 months (Fig. 1). She did not have any underlying diseases or a history of trauma prior to the appearance of the swelling. She complained of intermittent neuralgia on the cheek due to recent growth of the mass, which was about grade 3 according to the visual analogue scale. Other symptoms such as facial palsy, or sensory deficit were not observed. 


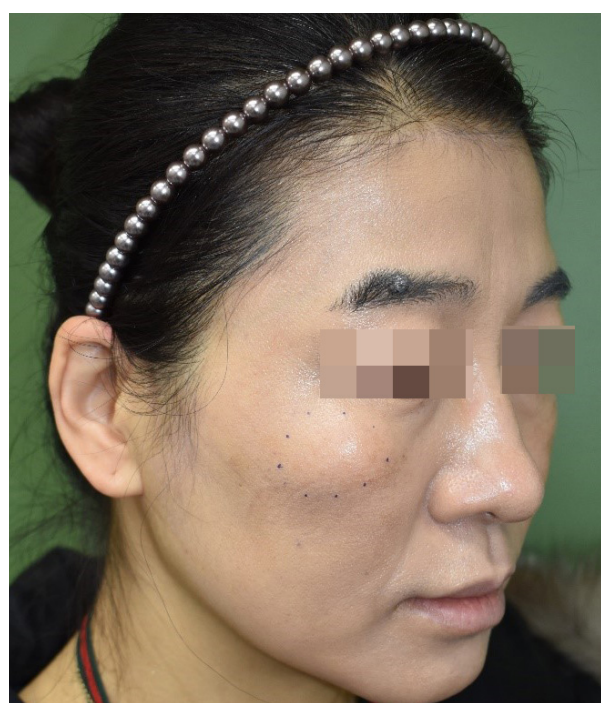

Fig. 1. Initial gross photograph of the mass. The mass was located in the right zygoma area, with a diameter of $2.2 \times 2.1 \mathrm{~cm}$.
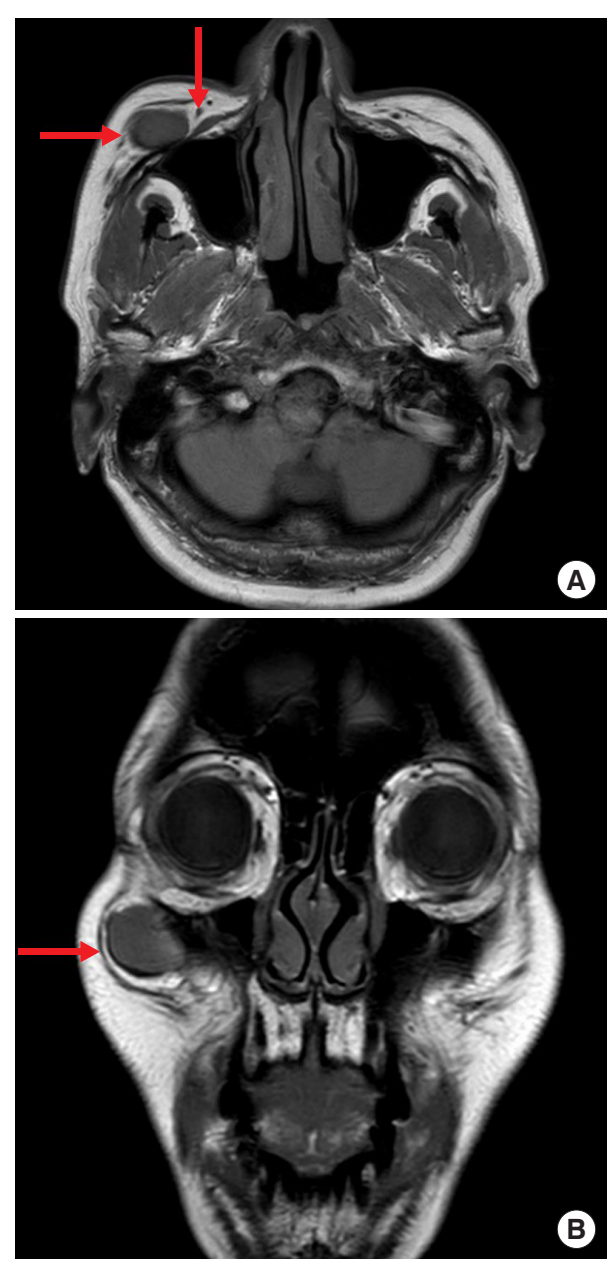

Fig. 2. Preoperative magnetic resonance imaging showing the mass, (A) The lobulated high-signal-intensity mass and feeding vessel (transverse facial artery) were shown on a T2-weighted image (red arrows). (B) It can be seen that the mass was separated from the orbicularis oculi in the coronal view of a T2-weighted image (red arrow).
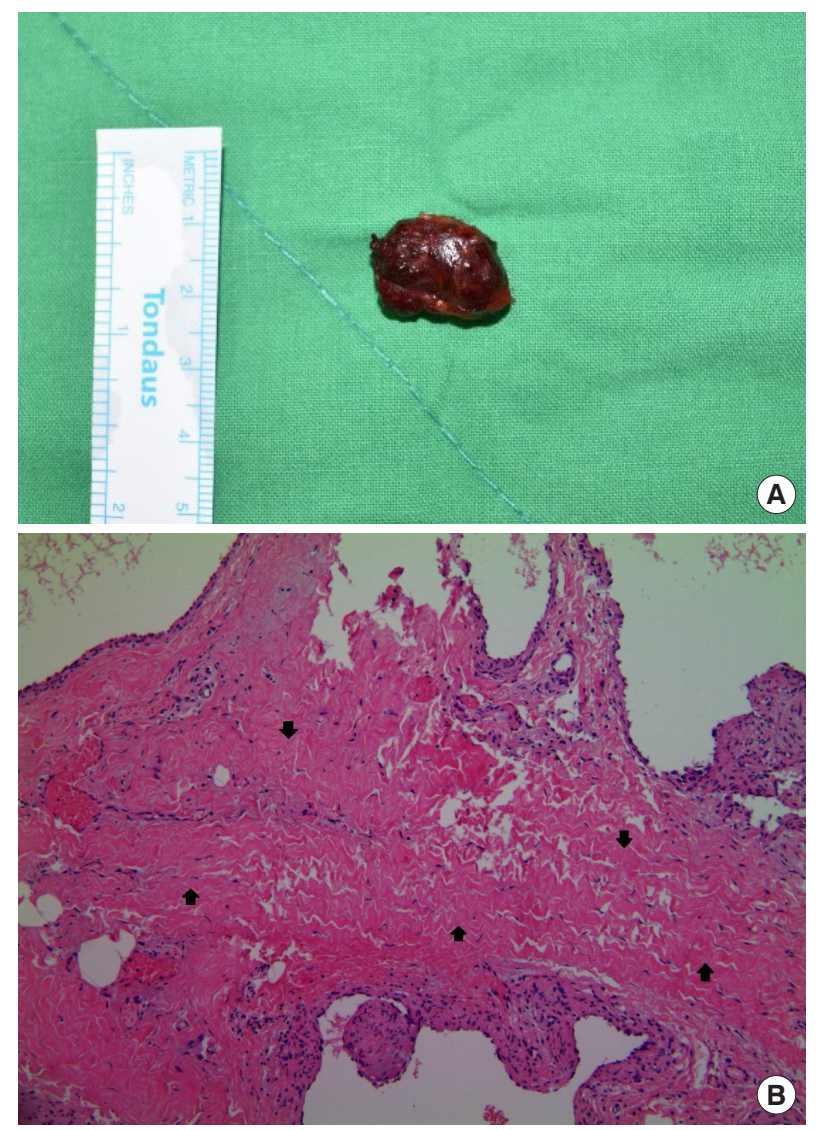

Fig. 3. Intraoperative gross photograph of the mass and photomicrograph of the specimen. (A) The diameter of the excised surgical specimen was about $2 \times 2 \mathrm{~cm}$. (B) The findings included an area of hemangioma infiltrating between the muscle fibers (black arrows, $\mathrm{H} \& \mathrm{E}, \times 100)$.

An ultrasonographic (US) examination revealed a well-marginated hypoechoic mass with suspicious compression of the underlying bone. US-guided fine-needle aspiration biopsy (FNAB) was performed, and the pathologic findings suggested that the specimen had several ectatic blood vessels, suggestive of IMH. Contrast-enhanced MRI showed a lobulated high-signal-intensity mass with heterogeneous strong enhancement and blood vessels presumed to be feeding vessels (Fig. 2A).

The patient underwent surgical resection under general anesthesia. Intraoperatively, the mass was firmly attached to the ZMi and zygoma body. As a result of slightly cutting the part of the muscle by electrocautery device and carefully peeling the soft tissue using peanut dissectors, the feeding vessel (the transverse facial artery) and adjacent nerve (the zygomaticofacial nerve) were not injured, and the main mass was completely excised (Fig. 3A). The histopathological findings included an area of capillary type of hemangioma infiltrating between the muscle fibers which were compatible with IMH (Fig. 3B).

A follow-up examination showed complete wound healing 
without facial palsy or muscle dysfunction 1 month postoperatively.

\section{DISCUSSION}

Hemangiomas and vascular malformations are benign neoplasms of blood vessels $[1,4]$. IMH is a special type of hemangioma involving the skeletal muscle that comprises only $0.8 \%$ of all hemangiomas [2]. Clinically, IMH presents as a localized swelling with smooth surface and incompressible borders. Due to overlying musculature, there are no relevant vascular findings such as pulsations, thrills, or bruits despite the vascular origin of the lesion. In rare cases, facial pain or headache may occur because of compression by the enlarging mass [5]. However, since IMH has no characteristic clinical symptoms, it is often misdiagnosed [6] and inappropriate surgical interventions are performed [7]. The differential diagnosis for masses of the zygomatic area includes lipoma, schwannoma, neurofibroma, and dermoid cyst $[3,6]$.

US is a good option for preoperative diagnosis, but the vascularity of IMH is often not evident on US [8]. US-guided FNAB does not play a decisive role in the diagnosis, as it only obtains bloody specimens due to the bleeding tendency of these lesions [7]. Salzman et al. [8] introduced a US-guided core needle biopsy $(\mathrm{CNB})$ protocol that was successfully performed for the preoperative diagnosis of IMHs, however; due to bleeding risk, it is controversial whether FNAB or CNB should be done for accurate diagnosis [8]. MRI seems to be a better imaging modality to evaluate IMH than computed tomography [4]. IMH presents a welldemarcated, bright lesion that has higher signal intensity in T2weighted images because of the stagnant blood in the vessels [2]. Angiography may be useful for preoperative embolization if a large feeding artery is adjacent to the mass, but is not routinely performed for the diagnosis of IMH [3]. In this case, although the transverse facial artery was confirmed as a feeding vessel through MRI (Fig. 2B), preoperative embolization was not performed after consultation with the patient and the intervention department about the possibility of remarkable changes of the mass.

Currently, the treatment of choice of IMH is surgical en bloc resection. Surgery is indicated for symptomatic IMHs causing facial pain or neuralgia, IMHs with a sudden increase in size, suspected malignant tumors, or IMHs that cause cosmetic problems [5]. The most important aspect in the surgical approach to head and neck tumors, especially those in the temple, zygoma, and preauricular area, is protection of the facial nerve branch [6]. Additional concerns from an aesthetic standpoint are hiding visible scar formation and compensation of volume loss after mass removal. In our case, a direct skin incision along relaxed skin tension lines was done with the patient's consent, and we performed complete resection without any injury of the branches and trunk of the facial nerve. The patient had no abnormality in facial expression postoperatively. Also, we considered to employ a fat graft intraoperatively, but she eventually refused because she wanted to make a decision based on the progress. She was satisfied with the result, and no serious complications were noted so far.

The ZMi is a muscle of facial expression, which is used in smiling by raising the upper lip upward and outward. It originates from the zygomatic bone immediately behind the zygomaticomaxillary suture, continues with the orbicularis oculi on the lateral surface of midface, and then inserts into the outer part of the upper lip $[9,10]$. Previous researchers have stated that it was challenging to distinguish between the ZMi and orbicularis oculi based on cadaveric studies, however; the difficulty of distinguishing between these muscles may depend on how the muscles are examined [9]. In this case, we confirmed preoperatively that the mass was separated from the orbicularis oculi on the coronal view of T2-weighted MRI (Fig. 2B). It was not easy to identify the origin of ZMi in the intraoperative field. However, we considered the vectors of the muscles in the midface; the muscle we examined ran at an oblique angle and inserted into the orbicularis oris muscle, so it must have been the ZMi. By slightly cutting the part of the muscle via electrocautery device that was in contact with the mass, we were able to approach to hemangioma located in the intramuscular level. Then, we split the muscle fibers for dissecting surrounding tissues and finally excised the lesion successfully. The severed ZMi was reattached using vicryl, and there were no concerns about the patient's facial expressions postoperatively. To our knowledge, this is the first report of an IMH in the $\mathrm{ZMi}$, and this report is expected to be helpful for the appropriate management of masses located in deep layers around the head and neck area.

In conclusion, head and neck IMH is a rare benign tumor that is difficult to diagnose. A proper radiological evaluation is required before choosing the surgical approach, and complete resection is the treatment of choice. Our case report may serve as a reference for head and neck IMH in the $\mathrm{ZMi}$, as it was successfully treated without nerve damage or muscle dysfunction. Long-term follow-up is recommended to determine whether there is recurrence or abnormality of facial expression.

\section{NOTES}

\section{Conflict of interest}

No potential conflict of interest relevant to this article was reported. 


\section{Ethical approval}

The study was approved by the Institutional Review Board of Hallym Sacred Heart Hospital (IRB No. 2021-02-016) and performed in accordance with the principles of the Declaration of Helsinki. Written informed consent was obtained.

\section{Patient consent}

The patient provided written informed consent for the publication and the use of her images.

\section{ORCID}

Sung Jae Wee https://orcid.org/0000-0002-2397-7450

Myong Chul Park https://orcid.org/0000-0002-0845-2672 Chan Min Chung https://orcid.org/0000-0002-2755-9440 Seung Wan Tak https://orcid.org/0000-0001-7744-5559

\section{Author contribution}

Conceptualization: SJW, MCP. Data curation: SJW, MCP. Formal analysis, methodology: SJW. Visualization: SJW, SWT. Investigation: SJW, CMC. Resources: SJW. Supervision: MCP, CMC, SWT. Writing - original draft: SJW. Writing - review \& editing: MCP, CMC, SWT.

\section{REFERENCES}

1. Kumar LKS, Kurien NM, Venugopal K, Nair PR, Mony V. Intramuscular hemangioma of the masseter muscle: a case report and review of literature. Int J Surg Case Rep 2016;26:209-16.

2. Lescura CM, de Andrade BAB, Bezerra KT, Agostini M, Ankha
MVA, de Castro F, et al. Oral intramuscular hemangioma: report of three cases. J Cutan Pathol 2019;46:603-8.

3. Aloyouny AY, Mehanny MS, Albagieh HN, Alfaleh WM, Mansour SM, Mobarak FA. Intramuscular hemangioma in the zygomaticus muscle: a rare case report presentation and diagnosis. Int J Surg Case Rep 2020;74:42-5.

4. Park H, Kim JS, Park H, Kim JY, Huh S, Lee JM, et al. Venous malformations of the head and neck: a retrospective review of 82 cases. Arch Plast Surg 2019;46:23-33.

5. Cho JK, Cha W, Sung MW. Intramuscular hemangioma in the anterior scalene muscle diagnosed by core needle biopsy. Clin Exp Otorhinolaryngol 2015;8:298-301.

6. Hwang JH, Lee DG, Sim HS, Kim KS, Lee SY. Intramasseteric schwannoma treated with facelift incision and retrograde facial nerve dissection. Arch Craniofac Surg 2019;20:388-91.

7. Righi S, Boffano P, Malve L, Rossi P, Zanardi F, Pateras D. Intramural perimasseteric hemangiomas of the inner cheek. J Craniofac Surg 2015;26:959-60.

8. Salzman R, Buchanan MA, Berman L, Jani P. Ultrasoundguided core-needle biopsy and magnetic resonance imaging in the accurate diagnosis of intramuscular haemangiomas of the head and neck. J Laryngol Otol 2012;126:391-4.

9. Youn KH, Park JT, Park DS, Koh KS, Kim HJ, Paik DJ. Morphology of the zygomaticus minor and its relationship with the orbicularis oculi muscle. J Craniofac Surg 2012;23:546-8.

10. Choi DY, Hur MS, Youn KH, Kim J, Kim HJ, Kim SS. Clinical anatomic considerations of the zygomaticus minor muscle based on the morphology and insertion pattern. Dermatol Surg 2014;40:858-63. 